\title{
The minimal length case of the Klein Gordon equation with hyperbolic cotangent potential using Nikivorof- Uvarof Method
}

\author{
Isnaini Lilis Elviyanti ${ }^{1}$, Ahmad Aftah Syukron ${ }^{2}$ \\ ${ }^{1,2}$ Physics Department, Ma'arif Nahdlatul Ulama University Kebumen \\ Email: ${ }^{1}$ isna.elviyanti@gmail.com
}

Received 10 January 2020, Revised 5 February 2020, Published 29 March 2020

\begin{abstract}
The case of minimal length is applied for the Klein Gordon equation with hyperbolic cotangent potential. The Klein Gordon equation for minimal length case is solved used to approximate solution. The energy eigenvalue and wave function are investigated by the Nikivorof-Uvarof method.
\end{abstract}

Keyword: Klein Gordon Equation, minimal length, hyperbolic cotangent potential

\section{Introduction}

In quantum mechanics based on the following deformed commutation relations between position and momentum operators in Heisenberg's uncertainty principle was called the minimal length. In the case of minimal length was occurring in position uncertainty and momentum uncertainty was influenced by a field. The general equation was given as (Sprenger, Piero Nicolini, \& Bleicher, 2012) and (Garay, 1994)

$$
[X, P] \geq i \hbar\left(1+\alpha_{M L}(\Delta P)^{2}\right)
$$

where $X$ was position, $P$ was the corresponding momentum and $\alpha_{M L}$ was a minimal length parameter that has value $0 \leq \alpha_{M L} \leq 1$. The minimal length case of the Klein Gordon equation had done by Bouaziz (Bouaziz, 2013) and Merad et al (Merad, Zeroual, \& Benzair, 2010).

The Klein Gordon equation is the study of behavior microscopic spin-zero particles in relativistic quantum mechanics (Ikhdair \& Hamzavi, 2012). The Klein Gordon equation has two conditions, which are the scalar potential was equal with vector potential $(S(r)=V(r))$ and the scalar potential was equal minus vector potential $(S(r)=-V(r))$. The Klein Gordon equation was given by,

$$
(E-V(r, \theta, \varphi))^{2} \Psi(r, \theta, \varphi)=\left[P^{2} c^{2}+\left(M_{o} c^{2}+S(r, \theta, \varphi)\right)^{2}\right] \Psi(r, \theta, \varphi)
$$


Where $E$ was relativistic energy and $M_{o}$ was rest mass. In these two conditions, Klein Gordon equation reduces to Schrodinger like equation thus a state solution was obtained using methods Supersymmetric Quantum Mechanics (SUSY) (Azizah, Suparmi, \& Cari, 2018), Nikivorof-Uvarof Method (NU) (Ikhdair S. M., 2011), and Asymptotic Iteration Method (AIM). (Elviyanti, Pratiwi, Suparmi, \& Cari, 2018), (Suparmi, Cari, \& Elviyanti, 2017), and (Cari, Suparmi, \& Elviyanti, 2017)

In this study, we solved the scalar potential was equal with the vector potential $(S(r)=V(r))$ condition of the Klein Gordon equation in minimal length case. The Klein-Gordon equation of the radial part solution for hyperbolic cotangent function potential has been studied. The Nikivorof-Uvarof method has been applied to obtain the relativistic energy and wave functions in the minimal length case. The work was organized as follows. In Section 2, the Klein Gordon equation for a minimal length case was introduced. In Section 3, we described briefly the Nikivorof-Uvarod method which was used to solve the Klein Gordon equation in minimal length case. In Section 4, the relativistic energy and wave function of the Klein Gordon equation was presented. The conclusion of the Klein Gordon equation with minimal length was presented in Section 5 .

\section{The Klein Gordon equation for minimal length case}

The minimal length equation in (1) can be written by

$$
\begin{gathered}
\hat{X}_{i}=\hat{x}_{i} \\
\hat{P}_{i}=\left(1+\alpha_{M L} \hat{p}^{2}\right) \hat{p}_{i}
\end{gathered}
$$

By setting $S(r, \theta, \varphi)=V(r, \theta, \varphi)$ in equation (2) and substituting equation (4) into equation (2) with $\hat{p}=-i \hbar \nabla, c=\hbar=1$ (natural unit), and $2 V(r) \rightarrow V(r)$, we obtained

$$
-\left(\Delta-2 \alpha_{M L} \Delta^{2}\right) \Psi(r)-\left(E^{2}-M_{o}^{2}-\left(E+M_{o}\right) V(r)\right) \Psi(r)=0
$$

By substituting a new wave function (Chabab, Batoul, Lahbas, \& Oulne, 2016) is $\Psi(r)=\left(1+2 \alpha_{M L}\right) \Phi(r)$ and using Binomial expansion, equation (5) became,

$$
\Delta \Phi(r)+\left(\begin{array}{l}
\left(E^{2}-M_{o}^{2}\right)-\left(E+M_{o}\right) V(r) \\
-2 \alpha_{M L}\left(E^{2}-M_{o}^{2}-\left(E+M_{o}\right) V(r)\right)^{2}
\end{array}\right) \Phi(r)=0
$$

The $\alpha_{M L}{ }^{2}$ from equation (6) that had very small value, so $\alpha_{M L}{ }^{2}$ it was ignored. By applying spherical Laplacian operator and using variable separation method, so we had a radial part,

$$
\left[\begin{array}{l}
\left(\frac{1}{r^{2}} \frac{\partial}{\partial r} r^{2} \frac{\partial}{\partial r}-\frac{L(L+1)}{r^{2}}\right)+\left(E^{2}-M_{o}^{2}-\left(E+M_{o}\right) V(r)\right) \\
-2 \alpha_{M L}\left(\left(E^{2}-M_{o}^{2}\right)^{2}-2\left(E^{2}-M_{o}^{2}\right)\left(E+M_{o}\right) V(r)+\left(E+M_{o}\right)^{2} V^{2}(r)\right)
\end{array}\right] U(r)=0
$$


Equation (7) was an approximate solution for the radial part of the Klein Gordon equation with the minimal length case.

\section{The Nikivorof-Uvarof Method}

The Nikivorof-Uvarof method is used to solve the second-order differential equation of hypergeometry type. The method can be written (Goudarzi \& Vahidi, 2011) and (Okon \& Popoola, 2015)

$$
\frac{d^{2} \psi}{d z^{2}}+\frac{\tilde{\tau}(z)}{\sigma(z)} \frac{d \psi}{d z}+\frac{\tilde{\sigma}(z)}{\sigma^{2}(z)} \psi=0
$$

Where $\tilde{\tau}(z)$ is first-order polynomial, $\sigma(z)$ and $\tilde{\sigma}(z)$ are second-order polynomial, as follow

$$
\begin{aligned}
& \sigma(z)=a x^{2}+b x+c \\
& \tilde{\sigma}(z)=f x^{2}+g x+h \\
& \tilde{\tau}(z)=d x+e
\end{aligned}
$$

By the general solution $\psi(z)=\phi(z) y(z)$ into equation, we obtain a hypergeometry type Goudarzi, Deta, ituen.

$$
\sigma \frac{\partial^{2} y}{\partial z^{2}}+\tau \frac{\partial y}{\partial z}+\lambda y=0
$$

The function $\phi(z)$ is a logarithmic derivative, as defined

$$
\frac{\phi^{\prime}}{\phi}=\frac{\pi}{\sigma}
$$

The function $\pi(z)$ is a polynomial with the parameter $z$. The function $\pi(z)$ and parameter $\lambda$ as follows Goudarzi, Deta, ituen),

$$
\begin{gathered}
\pi=\frac{\sigma^{\prime}(z)-\tilde{\tau}(z)}{2} \pm \sqrt{\left(\frac{\sigma^{\prime}(z)-\tilde{\tau}(z)}{2}\right)^{2}-\tilde{\sigma}(z)+k \sigma(z)} \\
\lambda=k+\pi^{\prime}
\end{gathered}
$$

The value of $\mathrm{k}$ is expression under the square root must be square of a polynomial, and then obtain a new eigenvalue equation,

$$
\lambda=\lambda_{n}=-n \tau^{\prime}-\frac{n(n-1)}{2} \sigma^{\prime \prime}
$$

Where $n=1,2,3, \ldots$ and $\tau=\tau+2 \pi$. By the equation and can get energy eigenvalue. The function $y(z)$ is the hypergeometry type can be solved by using Rodrigues relation,

$$
y_{n}(z)=\frac{C_{n}}{\rho(\mathrm{z})} \frac{d^{n}}{d z^{n}}\left(\sigma^{n}(z) \rho(\mathrm{z})\right)
$$

with $C_{n}$ is normalization constant and $\rho(\mathrm{z})$ is the weight function as follow,

$$
\frac{\partial(\sigma \rho)}{\partial z}=\tau(z) \rho(z)
$$


By the general solution $\psi(z)=\phi(z) y(z)$, can be obtained from the wave function of system Goudarzi, Deta, ituen).

\section{The Result and Discussion}

By applying the hyperbolic cotangent potential (cari) [24] was given as

$$
V(r)=V_{o} \operatorname{coth}(\beta r)
$$

where $V_{o}$ was constant of potential and $\omega$ was a range of potential. Then equation (17) and by the centrifugal approximation $\frac{1}{r^{2}}=\frac{\beta}{\sinh ^{2}(\beta r)}$ into equation (7), we obtained

$$
\left[\begin{array}{l}
\frac{d^{2} U(r)}{d r^{2}}-\left(L(L+1)+2 \alpha_{M L}\left(E+M_{o}\right)^{2} V_{o}^{2}\right) \operatorname{csch}^{2} \beta r U(r) \\
+\left(\begin{array}{l}
\left(E^{2}-M_{o}{ }^{2}\right)-2 \alpha_{M L}\left(E^{2}-M_{o}{ }^{2}\right)^{2}-2 \alpha_{M L}\left(E+M_{o}\right)^{2} V_{o}^{2} \\
+\left(4 \alpha_{M L}\left(E^{2}-M_{o}{ }^{2}\right)\left(E+M_{o}\right)-\left(E+M_{o}\right)\right) V_{o} \operatorname{coth} \beta r
\end{array}\right) U(r)
\end{array}\right]=0
$$

By setting coth $\beta r=s$ in equation (18), we have

$$
\left[\begin{array}{l}
\frac{d^{2} U(r)}{d s^{2}}+\frac{2 s\left(1-s^{2}\right)}{\left(1-s^{2}\right)^{2}} \frac{d U(r)}{d s}+\frac{\left(1-s^{2}\right)}{\left(1-s^{2}\right)^{2}} A_{\beta} U(r) \\
+\frac{s}{\left(1-s^{2}\right)^{2}} B_{\beta} U(r)+\frac{1}{\left(1-s^{2}\right)^{2}} C_{\beta} U(r)
\end{array}\right]=0
$$

With

$$
\begin{gathered}
A_{\beta}=\frac{\left(L(L+1)+2 \alpha_{M L}\left(E+M_{o}\right)^{2} V_{o}^{2}\right)}{\beta^{2}} \\
B_{\beta}=\frac{\left(4 \alpha_{M L}\left(E^{2}-M_{o}{ }^{2}\right)\left(E+M_{o}\right)-\left(E+M_{o}\right)\right) V_{o}}{\beta^{2}} \\
C_{\beta}=\frac{\left(\left(E^{2}-M_{o}{ }^{2}\right)-2 \alpha_{M L}\left(E^{2}-M_{o}{ }^{2}\right)^{2}-2 \alpha_{M L}\left(E+M_{o}\right)^{2} V_{o}^{2}\right)}{\beta^{2}}
\end{gathered}
$$

The equation (19) was a differential equation that had to be reduced to the NikivorofUvarof method equation type. From equation (19) can be parameters Nikivorof-Uvarof method, as follows

$$
\begin{gathered}
\tilde{\tau}(s)=-2 s \\
\sigma(s)=1-s^{2} \\
\tilde{\sigma}(s)=A_{\beta}\left(1-s^{2}\right)+B_{\beta} s+C_{\beta}
\end{gathered}
$$

By equation (12), we had

$$
\pi_{1,2}= \pm \sqrt{\left(A_{\beta}-k\right) s^{2}-B_{\beta} s+\left(k-A_{\beta}-C_{\beta}\right)}
$$

By setting $D=0$ or $b^{2}-4 a c=0$, the value of $\mathrm{k}$ was given 


$$
\begin{gathered}
k_{1}=\frac{\sqrt{C_{\beta}^{2}-B_{\beta}^{2}}-C_{\beta}}{2}+A_{\beta} \\
k_{2}=\frac{-\sqrt{{C_{\beta}{ }^{2}-B_{\beta}^{2}}^{2}}-C_{\beta}}{2}+A_{\beta}
\end{gathered}
$$

The equation (27) and (28) was inserted by equation (26), we had two conditions and one of these solutions satisfied bound state condition which is

$$
\pi_{1}=-\sqrt{\frac{C_{\beta}-\sqrt{C_{\beta}^{2}-B_{\beta}^{2}}}{2}}\left(s+\frac{-B_{\beta}}{C_{\beta}-\sqrt{C_{\beta}^{2}-B_{\beta}^{2}}}\right)
$$

Then by equation (13), we obtained

$$
\begin{gathered}
\tau_{1}=-2 s+2\left(-\sqrt{\frac{C_{\beta}-\sqrt{C_{\beta}^{2}-B_{\beta}^{2}}}{2}}\left(z+\frac{-B_{\omega \delta}}{C_{\beta}-\sqrt{C_{\beta}^{2}-B_{\beta}^{2}}}\right)\right) \\
\tau^{\prime}(s)=-2-2 \sqrt{\frac{C_{\beta}-\sqrt{C_{\beta}^{2}-B_{\beta}^{2}}}{2}}
\end{gathered}
$$

By applying equation (13)-(14) and (29)-(31), we had

$$
\left(E^{2}-M_{o}^{2}\right)=\left[\begin{array}{l}
\beta^{2}\left[\frac{A_{l \delta}+(1-n) \sqrt{C_{\omega \varepsilon}{ }^{2}-B_{\omega \delta}{ }^{2}}-2 n^{2}}{(1-n)}\right] \\
+2 \alpha_{M L}\left(E^{2}-M_{o}^{2}\right)^{2}+2 \alpha_{M L}\left(E+M_{o}\right)^{2} V_{o}^{2}
\end{array}\right]
$$

The equation (32) was the energy eigenvalue equation of the Klein Gordon equation for minimal length case. For calculating the wave function, we used a general solution $\psi(z)=\phi(z) y(z)$. By using equation (11) we obtained

$$
\phi(s)=\left(1-s^{2}\right)^{\frac{p_{1}}{2}}(1+s)^{\frac{B_{\beta}}{4 p_{1}}}(1-s)^{-\frac{B_{\beta}}{4 p_{1}}}
$$

The function $y(z)$ was found from the calculation of weight function in equation (16), so we had

$$
\rho(s)=\left(1-s^{2}\right)^{p_{1}}(1+s)^{\frac{B_{\beta}}{2 p_{1}}}(1-s)^{-\frac{B_{\beta}}{2 p_{1}}}
$$

So the function as follows

$$
y_{n}(s)=\left[\begin{array}{l}
C_{n}(1+s)^{-p_{1}+\frac{B_{\beta}}{2 p_{1}}}(1-s)^{-p_{1}-\frac{B_{\beta}}{2 p_{1}}} \\
\frac{d^{n}}{d s^{n}}\left(\left(1-s^{2}\right)(1+s)^{p_{1}+\frac{B_{\beta}}{2 p_{1}}}(1-s)^{p_{1}-\frac{B_{\beta}}{2 p_{1}}}\right)
\end{array}\right]
$$

with $p_{1}=\sqrt{\frac{C_{\beta}-\sqrt{C_{\beta}^{2}-B_{\beta}^{2}}}{2}}$. The equation (35) can be expressed Jacoby polynomial is given

$$
y_{n}(s)=(-1)^{n} 2^{n} n ! P_{n}^{\gamma, \delta}(s)=B_{n} P_{n}^{\gamma, \delta}(s)
$$

With 


$$
\begin{aligned}
& P_{n}^{\gamma \delta}=\frac{(-1)^{n}}{2^{n} n !}(1-z)^{-\gamma}(1+z)^{-\delta} \frac{d}{d z^{n}}(1-z)^{\gamma}(1+z)^{\delta}\left(1-z^{2}\right)^{n} \\
& \gamma=-p_{1}+\frac{B_{\beta}}{2 p_{1}} \\
& \delta=-p_{1}-\frac{B_{\beta}}{2 p_{1}}
\end{aligned}
$$

So we have

$$
\psi(s)=B_{n}\left((1+\operatorname{coth} \beta r)^{\frac{p_{1}}{2}+\frac{B_{\beta}}{4 p_{1}}}(1-\operatorname{coth} \beta r)^{\frac{p_{1}}{2}-\frac{B_{\beta}}{4 p_{1}}}\right) P_{n}^{\gamma, \delta}(\operatorname{coth} \beta r)
$$

$\mathrm{B}_{\mathrm{n}}$ is normalization constant. The equation (38) was wave function of the Klein Gordon equation in minimal length case using the Nikiforof-Uvarof method. For the example wave function $n=0, n=1$ and $n=2$, was given by

$$
\begin{aligned}
& \psi_{0}(s)=B_{0}\left((1+\operatorname{coth} \beta r)^{\frac{p_{1}}{2}+\frac{B_{\beta}}{4 p_{1}}}(1-\operatorname{coth} \beta r)^{\frac{p_{1}}{2}-\frac{B_{\beta}}{4 p_{1}}}\right) \\
& \psi_{1}(s)=\left[\begin{array}{l}
B_{1}\left((1+\operatorname{coth} \beta r)^{\frac{p_{1}}{2}+\frac{B_{\beta}}{4 p_{1}}}(1-\operatorname{coth} \beta r)^{\frac{p_{1}}{2}-\frac{B_{\beta}}{4 p_{1}}}\right) \\
\frac{1}{2}\left\{\left(1-\operatorname{coth}^{2} \beta r\right)\left(\gamma(1-\operatorname{coth} \beta r)^{1}-\delta(1+\operatorname{coth} \beta r)^{-1}\right)+2 \operatorname{coth} \beta r\right\}
\end{array}\right] \\
& \psi_{2}(s)=\left[\begin{array}{l}
B_{2}\left((1+\operatorname{coth} \beta r)^{\frac{p_{1}}{2}+\frac{B_{\beta}}{4 p_{1}}}(1-\operatorname{coth} \beta r)^{\frac{p_{1}}{2}}-\frac{B_{\beta}}{4 p_{1}}\right) \\
\frac{1}{8}\left[\begin{array}{l}
\left(1-\operatorname{coth}^{2} \beta r\right)^{2}\left\{\gamma(\gamma-1)(1-\operatorname{coth} \beta r)^{1}+\delta(1-\delta)(1+\operatorname{coth} \beta r)^{-1}\right\} \\
-2 s\left\{\begin{array}{l}
2 \delta \operatorname{coth} \beta r(1+\operatorname{coth} \beta r)^{-1} \\
+2 \delta \operatorname{coth} \beta r(1-\operatorname{coth} \beta r)^{-1}+\gamma \delta
\end{array}\right\}+8 \operatorname{coth}^{2} \beta r
\end{array}\right]
\end{array}\right]
\end{aligned}
$$

\section{Conclusion}

We investigated the Klein Gordon equation for the minimal length case. It influenced by hypergeometric cotangent potential using the Nikivorof-Uvarof method. NikiforovUvarof method was used to obtain the energy eigenvalue and wave functions. The energy eigenvalue of the Klein Gordon equation for minimal length case corresponding minimal length parameter and potential constant. The wave function was obtained in terms of the Jacobi polynomials.

\section{References}

Azizah, H., Suparmi, \& Cari. (2018). Analysis of Minimal Effects on the Relativistic Energy and Wave Function for the Exponential Type Potential Using Supersymmetric Quantum Mechanics. Journal of Physics: Conf. Series 997 , 012017.

Bouaziz, D. (2013). Klein-Gordon Equation with Coulomb Potential in the Presence of a Minimal Length. arXiv:1311.7405v1[quant-ph] . 
Cari, C., Suparmi, A., \& Elviyanti, I. L. (2017). Approximate Solution for the Minimal Length Case of the Klein Goron Equation for Trigonometric Cotangent Potential Using Asymptotic Iteration Method. Journal of Physics: Conf. Series 909.

Chabab, M., Batoul, A., Lahbas, A., \& Oulne, M. (2016). On gamma-rigid Regime of the Bohr-Mottelson Hamiltonian in the Presence of a Minimal Length. Physics Letters B 758 , 212-216.

Elviyanti, I. L., Pratiwi, N. B., Suparmi, A., \& Cari, C. (2018). The Application of Minimal Length in Klein-Gordon Equation with Hulthen Potential Using Asymptotic Iteration Method. Hindawi Advances in Mathematical Physics .

Garay, J. L. (1994). Quantum Gravity and Minimum Length. International Journal of Modern Physics A , 10(2), 145-165.

Goudarzi, H., \& Vahidi, V. (2011). Supersymmetric Approach for Eckart Potential Using the NU Method. Adv. Studies Theor. Phys. , 5(10), 469-576.

Ikhdair, S. M. (2011). Bound States of the Klein-Gordon for Exponential-Type Potential in D-Dimensions. Journal of Quantum Information Science , 2011(1), 73-86.

Ikhdair, S., \& Hamzavi, M. (2012). Effects of External Fields on a Two-Dimensional Klein-Gordon Particle Under Pseudo-harmonicosilator Interaction. Chin. Phys. $B, 21(11)$.

Merad, M., Zeroual, F., \& Benzair, H. (2010). Spinless Relativistic Particle in the Presence a Minimal Length. Electronic Journal of Theoritical Physics , 7(23), 41-56.

Okon, I. B., \& Popoola, O. (2015). Bound-State Solution of Schrodinger Equation with Hulthen plus Generalized Exponential Coulomb Potential Using NikiforovUvarov Method. International journal of Recent Adnvances in Physics (IJRAP) , 4(3).

Sprenger, M., Piero Nicolini, P., \& Bleicher, M. (2012). Physics on the smallest scales: an introduction to minimal length phenomenology. European Journal of Physics, 33, 853-862.

Suparmi, A., Cari, C., \& Elviyanti, I. L. (2017). Solution of Klein Gordon Equation fot Trigonometric Cotangent Potential in the Presence of a Minimal Length Using Asymptotic Iteration Method. Journal of Physics: Conf. Series 909. 